\title{
LOW MACH NUMBER LIMIT FOR VISCOUS COMPRESSIBLE FLOWS
}

\author{
RAPHAËL DANCHIN ${ }^{1}$
}

\begin{abstract}
In this survey paper, we are concerned with the zero Mach number limit for compressible viscous flows. For the sake of (mathematical) simplicity, we restrict ourselves to the case of barotropic fluids and we assume that the flow evolves in the whole space or satisfies periodic boundary conditions. We focus on the case of ill-prepared data. Hence highly oscillating acoustic waves are likely to propagate through the fluid. We nevertheless state the convergence to the incompressible Navier-Stokes equations when the Mach number $\epsilon$ goes to 0. Besides, it is shown that the global existence for the limit equations entails the global existence for the compressible model with small $\epsilon$. The reader is referred to [R. Danchin, Ann. Sci. Éc. Norm. Sup. (2002)] for the detailed proof in the whole space case, and to [R. Danchin, Am. J. Math. 124 (2002) 1153-1219] for the case of periodic boundary conditions.
\end{abstract}

Mathematics Subject Classification. 35B25, 35B40, 76N10.

Plenary lecture, Low Mach Number Flows Conference, June 21-25, 2004, Porquerolles, France.

\section{INTRODUCTION}

\subsection{Governing equations}

It is common sense that slightly compressible flows and incompressible flows do not differ much from one another. As a matter of fact, the so-called incompressible Navier-Stokes equations

$$
\left\{\begin{array}{l}
\partial_{t} u+u \cdot \nabla u-\nu \Delta u+\nabla \Pi=g \\
\operatorname{div} u=0
\end{array}\right.
$$

are often considered as relevant for describing slightly compressible liquids as water for instance. In this paper, we discuss to what extent this heuristic may be made rigorous from the mathematical viewpoint.

For the sake of simplicity, we restrict ourselves to the case of a barotropic fluid. The flow at point $(t, x)$ (throughout, it is understood that $t \in \mathbb{R}^{+}$denotes the time variable whereas $x \in \Omega \subset \mathbb{R}^{N}$ denotes the space variable) is thus characterized by its density $\rho=\rho(t, x) \in \mathbb{R}^{+}$and its velocity $u=u(t, x) \in \mathbb{R}^{N}$. The governing equations write:

$$
\left\{\begin{array}{l}
\partial_{t} \rho+\operatorname{div}(\rho u)=0 \\
\partial_{t}(\rho u)+\operatorname{div}(\rho u \otimes u)-\mu \Delta u-(\lambda+\mu) \nabla \operatorname{div} u+\nabla P=\rho f
\end{array}\right.
$$

Keywords and phrases. Low Mach number limit, compressible Navier-Stokes.

1 Laboratoire de Mathématiques et Applications, Université Paris 12, 61 avenue du Général de Gaulle, 94010 Créteil Cedex 10, France. danchin@univ-paris12.fr 
Above $f=f(t, x)$ stands for a given external force. The viscosity coefficients $\lambda$ and $\mu$ are assumed to be constant $^{1}$ and to satisfy $\mu>0$ and $\nu \stackrel{\text { def }}{=} \lambda+2 \mu>0$, a condition which insures ellipticity of the operator $-\mu \Delta-(\lambda+\mu) \nabla$ div and is satisfied for physical fluids. The barotropic hypothesis amounts to assuming that the pressure $P$ is a (suitably smooth) function of the density: $P=P(\rho)$.

In the present paper, we assume that the space variable $x$ belongs to the whole space $\mathbb{R}^{N}$ or to the periodic box $\mathbb{T}_{a}^{N}$ with period $2 \pi a_{i}$ in the $i$ th variable. We are concerned with the multi-dimensional case only: $N \geq 2$.

\subsection{The small Mach number regime}

In the small Mach number regime, the relevant time scale is of order $1 / \epsilon$ (where $\epsilon$ stands for the Mach number). Now, making the change of variable $t^{\epsilon}=\epsilon t$ and the change of unknown $(\rho, u)(t, x)=\left(\rho^{\epsilon}, \epsilon u^{\epsilon}\right)\left(t^{\epsilon}, x\right)$ the compressible Navier-Stokes equations rewrite:

$\left(\widetilde{N S C_{\epsilon}}\right)$

$$
\left\{\begin{array}{l}
\partial_{t} \rho^{\epsilon}+\operatorname{div}\left(\rho^{\epsilon} u^{\epsilon}\right)=0 \\
\partial_{t}\left(\rho^{\epsilon} u^{\epsilon}\right)+\operatorname{div}\left(\rho^{\epsilon} u^{\epsilon} \otimes u^{\epsilon}\right)-\mu \Delta u^{\epsilon}-(\lambda+\mu) \nabla \operatorname{div} u^{\epsilon}+\frac{\nabla P^{\epsilon}}{\epsilon^{2}}=\rho^{\epsilon} f^{\epsilon}
\end{array}\right.
$$

with $P^{\epsilon}=P\left(\rho^{\epsilon}\right)$. For notational convenience we dropped the exponent $\epsilon$ over the time variable. More details on the derivation of $\left(\widetilde{N S C_{\epsilon}}\right)$ may be found in the introduction of [20], or in [14].

Given a converging sequence of solutions $\left(\rho^{\epsilon}, u^{\epsilon}\right)$, what can be said about the system satisfied by the limit $(\rho, v)$ ? At the formal level, a necessary condition for the convergence of the momentum equation is that $\nabla P^{\epsilon} \rightarrow 0$ when $\epsilon$ goes to zero. Hence $\rho$ must be a (positive) constant $\bar{\rho}$. Then passing to the limit in the mass conservation equation, we get $\operatorname{div} u^{\epsilon} \rightarrow 0$. Coming back to the momentum equation, we conclude that $v$ must satisfy the incompressible Navier-Stokes equation

$$
\partial_{t} v+v \cdot \nabla v-\mu \Delta v+\nabla \Pi=f, \quad \operatorname{div} v=0,
$$

with initial datum $\mathcal{P} u^{0}$ (where $\mathcal{P}$ stands for the $L^{2}$ orthogonal projector over solenoidal vector-fields).

From now on, we make the stability assumption $P^{\prime}(\bar{\rho})>0$. Besides, performing a harmless change of variable, one can thus assume with no loss of generality that

$$
\bar{\rho}=1 \quad \text { and } \quad P^{\prime}(1)=1 .
$$

\subsubsection{The case of well-prepared data}

Passing to the limit in the momentum equation is easier if $\nabla P^{\epsilon}=\mathcal{O}\left(\epsilon^{2}\right)$, or, in other words, if we assume that $\rho^{\epsilon}-1=\mathcal{O}\left(\epsilon^{2}\right)$, and if besides $\operatorname{div} u^{\epsilon}=\mathcal{O}(\epsilon)$. Indeed, this entails that $\partial_{t} \rho^{\epsilon}$ and $\partial_{t} u^{\epsilon}$ are uniformly bounded so that the dangerous time oscillations cannot occur. Starting from this simple consideration, different authors have studied $\left(\widehat{N S C_{\epsilon}}\right)$ with data of the type $\rho_{0}^{\epsilon}=1+\epsilon^{2} \rho_{0,1}^{\epsilon}$ and $u_{0}^{\epsilon}=u_{0}+\epsilon u_{0,1}^{\epsilon}$ with $\operatorname{div} u_{0}=0$, and $\left(\rho_{0,1}^{\epsilon}, u_{0,1}^{\epsilon}\right)$ uniformly bounded in a suitable functional space ${ }^{2}$.

In the usual PDE'S terminology, such data are referred as well-prepared data. Indeed, they are well-prepared in the sense that they belong (up to lower order terms) to the kernel of the singular operator appearing in $\left(\widetilde{N S C}_{\epsilon}\right)$. Hence, they are unlikely to produce highly oscillating terms.

It is then possible to calculate an asymptotic expansion for $\left(\rho^{\epsilon}, u^{\epsilon}\right)$ in terms of powers of $\epsilon$. This approach (historically the first one in the framework of compressible fluids) has been followed by different authors: Klainerman and Majda in [18], Kreiss, Lorenz and Naughton in [19] and Hoff in [15] amongst others.

\footnotetext{
1 This assumption may be somewhat relaxed.

${ }^{2}$ We here take $f^{\epsilon} \equiv 0$ to simplify.
} 


\subsubsection{The case of ill-prepared data}

In the present paper, we make the weaker assumption that the initial density satisfies $\rho_{0}^{\epsilon}=1+\epsilon b_{0}^{\epsilon}$ and that $\left(b_{0}^{\epsilon}, u_{0}^{\epsilon}, f^{\epsilon}\right)$ tends to some limit in a suitable sense that we refrain from making precise since we are going to assume from now on (for simplicity only) that $b_{0}^{\epsilon}, u_{0}^{\epsilon}$ and $f^{\epsilon}$ are independent of $\epsilon$.

Denoting $\rho^{\epsilon}=1+\epsilon b^{\epsilon}$, it is found that $\left(b^{\epsilon}, u^{\epsilon}\right)$ satisfies

$$
\left\{\begin{array}{l}
\partial_{t} b^{\epsilon}+\frac{\operatorname{div} u^{\epsilon}}{\epsilon}=-\operatorname{div}\left(b^{\epsilon} u^{\epsilon}\right) \\
\partial_{t} u^{\epsilon}+u^{\epsilon} \cdot \nabla u^{\epsilon}-\frac{\mathcal{A} u^{\epsilon}}{1+\epsilon b^{\epsilon}}+\left(1+k\left(\epsilon b^{\epsilon}\right)\right) \frac{\nabla b^{\epsilon}}{\epsilon}=f \\
\left(b^{\epsilon}, u^{\epsilon}\right)_{\mid t=0}=\left(b_{0}, u_{0}\right)
\end{array}\right.
$$

with $\mathcal{A}:=\mu \Delta+(\lambda+\mu) \nabla \operatorname{div}$ and $k$ a smooth function satisfying $k(0)=0$.

The problem of passing to the limit in $\left(N S C_{\epsilon}\right)$ has been studied by different authors in the last decade. Roughly, two kinds of approach have been used.

The first one is based on the use of the following (formal) "energy equality":

$$
\begin{aligned}
\left\|\left(\sqrt{\rho^{\epsilon}} u^{\epsilon}\right)(t)\right\|_{L^{2}}^{2}+\int \pi^{\epsilon}(t, x) \mathrm{d} x+2 \int_{0}^{t}\left(\mu\left\|\nabla u^{\epsilon}\right\|_{L^{2}}^{2}+(\lambda+\mu)\left\|\operatorname{div} u^{\epsilon}\right\|_{L^{2}}^{2}\right) \mathrm{d} \tau= \\
\left\|\sqrt{\rho_{0}^{\epsilon}} u_{0}^{\epsilon}\right\|_{L^{2}}^{2}+\int \pi_{0}^{\epsilon}(x) \mathrm{d} x+2 \int_{0}^{t} \int \rho^{\epsilon} f^{\epsilon} \cdot u^{\epsilon}(\tau, x) \mathrm{d} x \mathrm{~d} \tau
\end{aligned}
$$

where $\pi^{\epsilon}$ stands for the "free energy per unit volume" and may be computed from $P^{\epsilon}$. For example, if $P^{\epsilon}=P\left(\rho^{\epsilon}\right)$ with $P(\rho)=a \rho^{\gamma}$, we have

$$
\pi^{\epsilon}=a \frac{\left(\rho^{\epsilon}\right)^{\gamma}-1-\gamma\left(\rho^{\epsilon}-1\right)}{\epsilon^{2} \gamma(\gamma-1)} .
$$

For fixed $\epsilon$, by combining (1) and very fine compactness arguments, the existence of global weak solutions with finite energy (i.e. finite left-hand side in (1)) has been stated by Lions in the viscous case for pressure laws of the type $P(\rho)=a \rho^{\gamma}$ with $\gamma$ large enough (see [21] for more precise statements).

Next, taking advantage of the uniform estimates provided by (1), it is possible to pass to the limit when $\epsilon$ goes to 0. This latter result has been stated by Lions, Desjardins, Grenier and Masmoudi in various contexts. The proof strongly depends on the boundary conditions. The reader may refer to [22] for the case of periodic boundary conditions, to [9] for the whole space and to [10] for the case of bounded domain with homogeneous Dirichlet conditions. Let us mention that Lions and Masmoudi have also proved some local weak convergence results regardless of the boundary conditions (see [23]).

From the practical viewpoint however, these nice and very general results of convergence for global weak solutions do not give much information in dimension $N \geq 3$. Indeed, owing to the use of compactness arguments, and to the fact that the uniqueness of the global weak solution of the limit system - the incompressible NavierStokes equation - has not been stated, convergence holds true only up to a subsequence. Of course, in dimension $N=2$ the global weak solution of the limit system is actually unique so that the whole sequence actually converges!

Let us also stress that this approach cannot be applied to elucidate the inviscid case.

Another possible approach for passing to the limit in $\left(N S C_{\epsilon}\right)$ amounts to assuming that the data are smooth enough so that the initial value problem $\left(N S C_{\epsilon}\right)$ is well-posed (i.e. to each data corresponds a unique solution on a small time interval, and small changes on the data induce small changes on the solution). As regards the study of the small Mach number limit in the framework of ill-prepared data, one can motion the works by Ukai, (see [29]), Schochet [28], Métivier, Schochet (see [26,27]) in the inviscid case, and the papers by Gallagher [12] and Hagström, Lorenz (see [14]) in the viscous case. 
In the present paper, we state the following results in the case $\mathbb{R}^{n}$ or $\mathbb{T}_{a}^{n}$ (see Thms. 4.2 and 5.2 for the full statements):

- For a suitable class of data, system $\left(N S C_{\epsilon}\right)$ has a unique solution on some non trivial time interval $\left[0, T_{\epsilon}\right]$.

- The time $T_{\epsilon}$ may be chosen so that $\liminf _{\epsilon \rightarrow 0} T_{\epsilon} \geq T$ where $T$ denotes the lifespan of the solution $v$ to the incompressible Navier-Stokes equations ${ }^{3}$ :

$(N S I)$

$$
\left\{\begin{array}{l}
\partial_{t} v+\mathcal{P}(v \cdot \nabla v)-\mu \Delta v=\mathcal{P} f \\
v_{\mid t=0}=\mathcal{P} u_{0}
\end{array}\right.
$$

In particular, if $T=+\infty$ then also $T_{\epsilon}=+\infty$ for small $\epsilon$.

- When $\epsilon$ goes to 0 , the incompressible part $\mathcal{P} u^{\epsilon}$ of $u^{\epsilon}$ tends strongly to $v$ in an appropriate functional space whereas the gradient part $\mathcal{Q} u^{\epsilon}$ of $u^{\epsilon}$, and $b^{\epsilon}$ tend to 0 in a weaker sense (which strongly depends on the boundary conditions, the periodic case being worse than the whole space case).

The whole proof of these results (which is quite long and technical) may be found in [6,7]. In the present survey, we skip the details in order to stay as far as possible at the elementary level.

\section{WELL-POSEDNESS RESULTS FOR COMPRESSIBLE FLOWS}

\subsection{A good functional framework}

Finding a good functional framework is one of the key points in the study of slightly compressible fluids. The difficulty lies in the fact that we have to exhibit a functional framework which suits both $\left(N S C_{\epsilon}\right)$ and the limit system $(N S I)$, despite the fact that $\left(N S C_{\epsilon}\right)$ is of hyperbolic/parabolic type whereas $(N S I)$ is definitely parabolic.

Let us first focus on the incompressible Navier-Stokes equations which have been more studied than $\left(N S C_{\epsilon}\right)$. It is well known that a good functional framework for $(N S I)$ must be related to its scaling invariance. More precisely, observing that the transformation

$$
v(t, x) \longrightarrow \lambda v\left(\lambda^{2} t, \lambda x\right)
$$

does not affect (NSI) (provided the data have been modified accordingly), one can guess that an appropriate functional space for (NSI) must have a norm which is invariant (up to an irrelevant constant) for all $\lambda>0$ by the above transformation. This induces to take initial data in a functional space invariant by $v_{0}(x) \longrightarrow \lambda v_{0}(\lambda x)$ and to show well-posedness in a functional space $E$ which is critical insofar as its norm is invariant by (2). It turns out that once well-posedness has been stated in a critical space $E$, it holds also true in any "reasonable" subspace $E^{\prime}$ such that $E^{\prime} \hookrightarrow E$.

The first example of solving (NSI) in a critical space has been exhibited by Fujita and Kato in 1964 (see [11]). There, the initial velocity belongs to the homogeneous Sobolev space $\dot{H}^{\frac{N}{2}-1}\left(\mathbb{R}^{N}\right)$ and well-posedness is stated in a subspace of $\mathcal{C}\left([0, T] ; \dot{H}^{\frac{N}{2}-1}\left(\mathbb{R}^{N}\right)\right)$. Since then, well-posedness has been stated in a plethora of critical spaces: Lebesgue space $L^{N}\left(\mathbb{R}^{N}\right)$ (see [16]), Besov spaces $\dot{B}_{p, r}^{\frac{N}{p}-1}$ (see [2]) etc.

Fortified by these results on the incompressible model, one can guess that the initial velocity $u_{0}$ for $\left(N S C_{\epsilon}\right)$ must belong to a space whose norm is invariant by $u_{0}(x) \longrightarrow \lambda u_{0}(\lambda x)$. We expect scaling considerations to help us to find the relevant space for the density. Now, we notice that if the pressure term is neglected in $\left(N S C_{\epsilon}\right)$, then the transformation

$$
\left(b^{\epsilon}(t, x), u^{\epsilon}(t, x)\right) \longrightarrow\left(b^{\epsilon}\left(\lambda^{2} t, \lambda x\right), \lambda u^{\epsilon}\left(\lambda^{2} t, \lambda x\right)\right)
$$

does not affect $\left(N S C_{\epsilon}\right)$.

\footnotetext{
${ }^{3}$ Remind that the operator $\mathcal{P}$ stands for the $L^{2}$ projector on divergence-free vector-fields.
} 
Hence, choosing data $\left(b_{0}, u_{0}\right)$ in a critical functional space $E \times F$ whose norm is invariant by

$$
\left(b_{0}(x), u_{0}(x)\right) \longrightarrow\left(b_{0}(\lambda x), \lambda u_{0}(\lambda x)\right)
$$

seems appropriate.

Note that it roughly means that $u_{0}$ and $\nabla b_{0}$ must have the same regularity. If we restrict ourselves to Sobolev spaces, this leads to the assumption that $u_{0}$ and $\nabla b_{0}$ are in $\dot{H}^{\frac{N}{2}-1}\left(\mathbb{R}^{N}\right)$.

\subsection{Existence of solutions with critical regularity}

Whether local well-posedness may be proved for $u_{0}$ and $\nabla b_{0}$ in $\dot{H}^{\frac{N}{2}-1}\left(\mathbb{R}^{N}\right)$ is unclear. The main reason why is that assuming $\nabla b_{0} \in \dot{H}^{\frac{N}{2}-1}\left(\mathbb{R}^{N}\right)$ does not provide any control on the $L^{\infty}$ norm of $b$ so that the appearance of vacuum in the flow cannot be excluded.

At least for small time, this trouble may be avoided if one supposes that $b_{0}$ belongs to a slightly smaller space which is continuously embedded in $L^{\infty}$. If one wishes to keep the scaling invariance, one can assume that $b_{0}$ belongs to the homogeneous Besov $\dot{B}_{2,1}^{\frac{N}{2}}$ which satisfies $\dot{B}_{2,1}^{\frac{N}{2}} \hookrightarrow L^{\infty}$ and whose norm is invariant by dilation.

We here recall that for $|s| \leq N / 2$, homogeneous Besov spaces $\dot{B}_{2,1}^{s}$ may be defined as the completion of smooth compactly supported functions for the norm

$$
\|z\|_{\dot{B}_{2,1}^{s}} \stackrel{\text { def }}{=} \sum_{q \in \mathbb{Z}} 2^{q s}\left\|\Delta_{q} z\right\|_{L^{2}}
$$

where $\left(\Delta_{q}\right)_{q \in \mathbb{Z}}$ stands for a homogeneous dyadic partition in Fourier variables, that is $\mathcal{F}\left(\Delta_{q} z\right)(\xi)=\varphi\left(2^{-q} \xi\right) \mathcal{F} z(\xi)$ for some smooth $\varphi$ supported in an annulus and such that $\sum_{q \in \mathbb{Z}} \varphi\left(2^{-q} \xi\right)=1$ whenever $\xi \neq 0$.

Remark that

$$
\|z\|_{\dot{H}^{s}} \approx\left(\sum_{q \in \mathbb{Z}} 2^{2 q s}\left\|\Delta_{q} z\right\|_{L^{2}}^{2}\right)^{\frac{1}{2}} .
$$

Hence $\dot{B}_{2,1}^{s} \hookrightarrow \dot{H}^{s}$. The spaces $\dot{B}_{2,1}^{s}$ and $\dot{H}^{s}$ are very close to one another though.

In $[3,5]$, we prove that $\left(N S C_{\epsilon}\right)$ with $\epsilon=1$ are well-posed in critical Besov spaces ${ }^{4}$. In particular, we get the following result:

Theorem 2.1. Assume that $b_{0}$ belongs to $\dot{B}_{2,1}^{\frac{N}{2}} \cap \dot{B}_{2,1}^{\frac{N}{2}-1}$ (with small norm), that $u_{0}$ belongs to $\dot{B}_{2,1}^{\frac{N}{2}-1}$ and that $f \in L^{1}\left(0, T ; \dot{B}_{2,1}^{\frac{N}{2}-1}\right)$. Then $\left(N S C_{1}\right)$ has a unique local solution $(b, u)$ such that

$$
\begin{aligned}
& b \in \mathcal{C}\left([0, T] ; \dot{B}_{2,1}^{\frac{N}{2}} \cap \dot{B}_{2,1}^{\frac{N}{2}-1}\right) \cap L^{2}\left(0, T ; \dot{B}_{2,1}^{\frac{N}{2}}\right), \\
& u \in \mathcal{C}\left([0, T] ; \dot{B}_{2,1}^{\frac{N}{2}-1}\right) \cap L^{1}\left(0, T ; \dot{B}_{2,1}^{\frac{N}{2}}+1\right) .
\end{aligned}
$$

Besides, if $\left\|u_{0}\right\|_{\dot{B}_{2,1}^{2}-1}$ is small compared to $\min (\mu, \lambda+2 \mu)$ then $T=+\infty$.

Remark 2.2. By performing a change of variable, system $\left(N S C_{\epsilon}\right)$ may be shown to be equivalent to $\left(N S C_{1}\right)$. Hence a similar statement holds true for $\left(N S C_{\epsilon}\right)$.

Remark 2.3. As expected, one can prove that more regular data yield more regular solutions.

\footnotetext{
${ }^{4}$ See [8] for the proof of uniqueness in dimension $N=2$.
} 


\section{The LINEARIZED EQUATIONS}

Investigating the linearized equations at $\left(b^{\epsilon}, u^{\epsilon}\right)=(0,0)$ is a key step in the study of the incompressible limit for $\left(N S C_{\epsilon}\right)$.

Since system $\left(N S C_{\epsilon}\right)$ writes

$$
\left\{\begin{array}{l}
\partial_{t} b^{\epsilon}+\frac{\operatorname{div} u^{\epsilon}}{\epsilon}=-\operatorname{div}\left(b^{\epsilon} u^{\epsilon}\right) \\
\partial_{t} u^{\epsilon}+u^{\epsilon} \cdot \nabla u^{\epsilon}-\frac{\mathcal{A} u^{\epsilon}}{1+\epsilon b^{\epsilon}}+\left(1+k\left(\epsilon b^{\epsilon}\right)\right) \frac{\nabla b^{\epsilon}}{\epsilon}=f
\end{array}\right.
$$

with $k$ a smooth function such that $k(0)=0$ and $\mathcal{A}=\mu \Delta+(\lambda+\mu) \nabla \operatorname{div}$, the linearized equations about $(0,0)$ read

$$
\left\{\begin{array}{l}
\partial_{t} \dot{b}+\frac{\operatorname{div} \dot{u}}{\epsilon}=\dot{F} \\
\partial_{t} \dot{u}-\mathcal{A} \dot{u}+\frac{\nabla \dot{b}}{\epsilon}=\dot{G}
\end{array}\right.
$$

For a more accurate analysis of this system, we shall decompose the second equation on solenoidal and gradientlike vector-fields. This may be done by introducing the $L^{2}$ projector on solenoidal vector-fields $\mathcal{P}$ and its conjugate projector ${ }^{5} \mathcal{Q} \stackrel{\text { def }}{=} \operatorname{Id}-\mathcal{P}$.

Denoting $\nu \stackrel{\text { def }}{=} \lambda+2 \mu$, and applying $\mathcal{P}$ and $\mathcal{Q}$ to the last line of the above linear system, we get

$$
\left\{\begin{array}{l}
\partial_{t} \dot{b}+\frac{\operatorname{div} \mathcal{Q} \dot{u}}{\epsilon}=\dot{F} \\
\partial_{t} \mathcal{Q} \dot{u}-\nu \Delta \mathcal{Q} \dot{u}+\frac{\nabla \dot{b}}{\epsilon}=\mathcal{Q} \dot{G}, \\
\partial_{t} \mathcal{P} \dot{u}-\mu \Delta \mathcal{P} \dot{u}=\mathcal{P} \dot{G} .
\end{array}\right.
$$

On the one hand $\mathcal{P} \dot{u}$ satisfies a mere heat equation with constant diffusion coefficient. On the other hand, there is a coupling between the first two equations through the singular first order terms $\epsilon^{-1} \operatorname{div} \dot{\mathcal{Q}} u$ and $\epsilon^{-1} \nabla \dot{b}$.

Introducing $\dot{d} \stackrel{\text { def }}{=}|D|^{-1} \operatorname{div} \dot{u}$ (where $|D|^{\alpha}$ stands for the Fourier multiplier $|\xi|^{\alpha}$ ) so that $\mathcal{Q} \dot{u}=\nabla|D|^{-1} \dot{d}$, the coupling between $\dot{b}$ and $\mathcal{Q} \dot{u}$ may be described by the following baby system:

$$
\left\{\begin{array}{l}
\partial_{t} \dot{b}+\frac{|D| \dot{d}}{\epsilon}=\dot{F} \\
\partial_{t} \dot{d}-\nu \Delta \dot{d}-\frac{|D| \dot{b}}{\epsilon}=\dot{G}
\end{array}\right.
$$

By performing a Fourier transform with respect to the space variables, the above model may be solved explicitly. Indeed, in Fourier variables, $\left(B M_{\epsilon}\right)$ reads

$$
\frac{\mathrm{d}}{\mathrm{d} t}\left(\begin{array}{c}
\mathcal{F} \dot{b} \\
\mathcal{F} \dot{d}
\end{array}\right)(t, \xi)=\underbrace{\left(\begin{array}{ll}
0 & -\epsilon^{-1}|\xi| \\
\epsilon^{-1}|\xi| & -\nu|\xi|^{2}
\end{array}\right)}_{A^{\epsilon}(\xi)}\left(\begin{array}{l}
\mathcal{F} \dot{b}(t, \xi) \\
\mathcal{F} \dot{d}(t, \xi)
\end{array}\right)+\left(\begin{array}{c}
\mathcal{F} \dot{F}(t, \xi) \\
\mathcal{F} \dot{G}(t, \xi)
\end{array}\right) .
$$

By computing the eigenvalues of the matrix $A^{\epsilon}(\xi)$, we discover that the system behaves quite differently at low and high frequencies.

${ }^{5}$ In $\mathbb{R}^{N}$ or $\mathbb{T}_{a}^{N}, \mathcal{Q}$ coincides with the 0 -th order pseudo-differential operator $-\nabla \operatorname{div}(-\Delta)^{-1}$ whose vector-valued symbol is $\xi|\xi|^{-2} \xi$. 
The low frequency regime: for $\nu \epsilon|\xi|<2$, the matrix $A^{\epsilon}(\xi)$ has two complex conjugate eigenvalues with negative real part:

$$
\lambda^{ \pm}(\xi)=-\frac{\nu|\xi|^{2}}{2}\left(1 \pm i \sqrt{\frac{4}{\epsilon^{2} \nu^{2}|\xi|^{2}}-1}\right)
$$

Hence in the asymptotic $\nu \epsilon|\xi| \rightarrow 0$, we have

$$
\lambda \pm(\xi) \sim-\nu \frac{|\xi|^{2}}{2} \mp i \frac{|\xi|}{\epsilon}
$$

In the low frequency regime, we thus expect the pseudo-differential operator $A^{\epsilon}(D)$ to behave like $-\frac{\nu}{2} \Delta \mp i \frac{|D|}{\epsilon}$. We conclude that at low frequencies the semi-group generated by $A^{\epsilon}(D)$ should inherit from the properties of the heat equation with diffusion $\frac{\nu}{2}$ and from the ones of the (half) wave equation with velocity $\epsilon^{-1}$.

The high frequency regime: for $\nu \epsilon|\xi|>2$, the matrix $A^{\epsilon}(\xi)$ has two negative eigenvalues, namely

$$
\lambda^{ \pm}(\xi)=-\frac{\nu|\xi|^{2}}{2}\left(1 \pm \sqrt{1-\frac{4}{\epsilon^{2} \nu^{2}|\xi|^{2}}}\right)
$$

In the asymptotic $\nu \epsilon|\xi| \rightarrow+\infty$, we have

$$
\lambda^{+}(\xi) \sim-\nu|\xi|^{2} \text { and } \quad \lambda^{-}(\xi) \sim-\frac{1}{\epsilon^{2} \nu}
$$

Hence, system $\left(B M_{\epsilon}\right)$ has one parabolic mode with diffusion $\nu$ (a further computation shows that this mode tends to be collinear to $\dot{d}$ ), and one damped mode with coefficient $\frac{1}{\epsilon^{2} \nu}$ (which tends to be collinear to $\dot{b}$ ).

According to this rough analysis, the tentative conclusion is that the velocity $\dot{u}$ has a parabolic behaviour. The behaviour of $\dot{b}$ is more complex: parabolic for low frequencies and damped for high frequencies. One also has to keep in mind that both $\dot{u}$ and $\dot{b}$ benefit from highly oscillating properties at low frequency, a regime which tends to overrun high frequencies when $\epsilon$ goes to $0 \ldots$

By combining the above heuristics with a few calculation, one eventually gets the following estimate for all $s \in \mathbb{R}$ and $t \in \mathbb{R}^{+}$and some universal constant $C$ :

$$
\begin{aligned}
\|\dot{b}(t)\|_{\widetilde{B}_{\epsilon \nu}^{s, \infty}}+\|\dot{u}(t)\|_{\dot{B}_{2,1}^{s-1}}+\int_{0}^{t}\left(\nu\|\dot{b}\|_{\widetilde{B}_{\epsilon \nu}^{s, 1}}+\min (\mu, \nu)\|\dot{u}\|_{\dot{B}_{2,1}^{s+1}}\right) \mathrm{d} \tau \leq \\
C\left(\left\|\dot{b}_{0}\right\|_{\widetilde{B}_{\epsilon \nu}^{s, \infty}}+\left\|\dot{u}_{0}\right\|_{\dot{B}_{2,1}^{s-1}}+\int_{0}^{t}\left(\nu\|\dot{F}\|_{\widetilde{B}_{\epsilon \nu}^{s, \infty}}+\min (\mu, \nu)\|\dot{G}\|_{\dot{B}_{2,1}^{s-1}}\right) \mathrm{d} \tau\right) .
\end{aligned}
$$

Above, $\|z\|_{\widetilde{B}_{\alpha}^{\sigma, 1}}$ and $\|z\|_{\widetilde{B}_{\alpha}^{\sigma, \infty}}$ stand for hybrid Besov norms defined as follows:

$$
\|z\|_{\widetilde{B}_{\alpha}^{\sigma, 1}} \stackrel{\text { def }}{=} \sum_{q \in \mathbb{Z}} 2^{q \sigma} \min \left(\alpha^{-1}, 2^{q}\right)\left\|\Delta_{q} z\right\|_{L^{2}}, \quad\|z\|_{\widetilde{B}_{\alpha}^{\sigma, \infty}} \stackrel{\text { def }}{=} \sum_{q \in \mathbb{Z}} 2^{q \sigma} \max \left(\alpha, 2^{-q}\right)\left\|\Delta_{q} z\right\|_{L^{2}} .
$$

Note that $\|\cdot\|_{\widetilde{B}_{\epsilon \nu}^{s, 1}}$ is equivalent to $\|\cdot\|_{\dot{B}_{2,1}^{s+1}}$ at low frequencies and to $(\epsilon \nu)^{-1}\|\cdot\|_{\dot{B}_{2,1}^{s}}$ at high frequencies whereas $\|\cdot\|_{\widetilde{B}_{\epsilon \nu}^{s, \infty}}$ is equivalent to $\|\cdot\|_{\dot{B}_{2,1}^{s-1}}$ at low frequencies and to $\epsilon \nu\|\cdot\|_{\dot{B}_{2,1}^{s}}$ at high frequencies. In both cases, the cut-off frequency between the two regimes is at $|\xi| \sim(\epsilon \nu)^{-1}$.

Remark 3.1. Hybrid Sobolev spaces $\widetilde{H}_{\epsilon \nu}^{s, 1}$ and $\widetilde{H}_{\epsilon \nu}^{s, \infty}$ may be defined in a similar way. It is then easy to state a similar estimate as (3) in the Sobolev framework (see [6]). 
Remark 3.2. Inequality (3) provides us with uniform estimates in $\mathcal{C}\left([0, T] ; \dot{B}^{s-1}\right) \cap L^{2}\left(0, T ; \dot{B}^{s}\right)$ for $(\dot{b}, \dot{u})$. Note however that we did not take advantage of the oscillating properties of $\left(B M_{\epsilon}\right)$ at low frequencies. This is due to the use of $L^{2}$ type estimates which kills the $i|D| / \epsilon$ part of the operator $A^{\epsilon}(D)$ at low frequency. In the next two sections, we shall see how to use those oscillating properties to pass to the limit in $\left(N S C_{\epsilon}\right)$.

\section{The INCOMPRESSIBle Limit IN THE CASE OF THE WHOLE SPACE}

\subsection{The heuristic}

The basic idea is that the highly oscillating acoustic waves go at infinity with the high speed $\epsilon^{-1}$ and polynomial decay ${ }^{6}$. This fact has been used before by S. Ukai in the inviscid case (see [29]) and in the viscous case by B. Desjardins and E. Grenier in the framework of global weak solutions (see [9]).

More concretely, proving that the compressible part $\left(b^{\epsilon}, \mathcal{Q} u^{\epsilon}\right)$ of the solution tends to zero relies on dispersive estimates of Strichartz type for the equation of acoustics:

$$
\left\{\begin{array}{l}
\partial_{t} \dot{b}+\frac{|D| \dot{d}}{\epsilon}=\dot{F}, \\
\partial_{t} \dot{d}-\frac{|D| \dot{b}}{\epsilon}=\dot{G} .
\end{array}\right.
$$

Let us mention in passing that Strichartz estimates have been discovered by Strichartz (see the original paper [28]) and have been extensively used lastly for dispersive equations such as the Schrödinger or the wave equation (see e.g. $[13,17]$ ).

Stating dispersive estimates for $\left(W_{\epsilon}\right)$ requires the use of Besov norms of type $L^{p}$ :

$$
\|z\|_{\dot{B}_{p, 1}^{\sigma}} \stackrel{\text { def }}{=} \sum_{q \in \mathbb{Z}} 2^{q \sigma}\left\|\Delta_{q} z\right\|_{L^{p}} \quad \text { if } \quad 1 \leq p<\infty, \quad \text { and } \quad\|z\|_{\dot{B}_{\infty, 1}^{\sigma}} \stackrel{\text { def }}{=} \sup _{q \in \mathbb{Z}} 2^{q \sigma}\left\|\Delta_{q} z\right\|_{L^{\infty}}
$$

Proposition 4.1. Let $(\dot{b}, \dot{d})$ be a solution to $\left(W_{\epsilon}\right)$. Then we have the inequality

$$
\|(\dot{b}, \dot{d})\|_{L_{T}^{r}\left(\dot{B}_{p, 1}^{\left.s+N\left(\frac{1}{p}-\frac{1}{2}\right)+\frac{1}{r}\right)}\right.} \leq C \epsilon^{\frac{1}{r}}\left(\left\|\left(\dot{b}_{0}, \dot{d}_{0}\right)\right\|_{\dot{B}_{2,1}^{s}}+\|(\dot{F}, \dot{G})\|_{L_{T}^{1}\left(\dot{B}_{2,1}^{s}\right)}\right)
$$

for some constant $C=C_{r, p, N}$ whenever

$$
p \geq 2, \quad \frac{2}{r} \leq \min \left(1,(N-1)\left(\frac{1}{2}-\frac{1}{p}\right)\right) \quad \text { and } \quad(r, p, N) \neq(2, \infty, 3)
$$

Example. For data $\left(b_{0}, u_{0}\right)$ in $\dot{B}_{2,1}^{\frac{N}{2}-\frac{1}{2}}$ and in the case where dimension is $N \geq 4$, one can choose $p=\infty$ and $r=2$. As we have the embedding $\dot{B}_{\infty, 1}^{0} \hookrightarrow L^{\infty}$, one gets

$$
\left\|\left(b^{\epsilon}, d^{\epsilon}\right)\right\|_{L_{T}^{2}\left(L^{\infty}\right)} \leq C \epsilon^{\frac{1}{2}}\left(\left\|\left(b_{0}, d_{0}\right)\right\|_{\dot{B}_{2,1}^{\frac{N}{2}-\frac{1}{2}}}+\|(F, G)\|_{L_{T}^{1}\left(\dot{B}_{2,1}^{\frac{N}{2}-\frac{1}{2}}\right)}\right) .
$$

Of course the above proposition also provides some decay in $\epsilon$ in the more physical cases where $N=2,3$.

\footnotetext{
${ }^{6}$ Note that this heuristic would be false in dimension $N=1$.
} 


\subsection{Results of convergence}

Let us first state a result of convergence in high dimension:

Theorem 4.2. Let $N \geq 3, \alpha \in(0,1 / 2)$ and $T \in(0,+\infty]$. Assume that $b_{0} \in \dot{B}_{2,1}^{\frac{N}{2}-1} \cap \dot{B}_{2,1}^{\frac{N}{2}+\alpha}, u_{0} \in \dot{B}_{2,1}^{\frac{N}{2}-1} \cap \dot{B}_{2,1}^{\frac{N}{2}+\alpha-1}$ and $f \in L^{1}\left(\mathbb{R}^{+} ; \dot{B}_{2,1}^{\frac{N}{2}-1} \cap \dot{B}_{2,1}^{\frac{N}{2}-1+\alpha}\right)$, and that system

$$
\left\{\begin{array}{l}
\partial_{t} v+v \cdot \nabla v-\mu \Delta v+\nabla \Pi=f, \quad \operatorname{div} v=0 \\
v_{\mid t=0}=\mathcal{P} u_{0}
\end{array}\right.
$$

has a unique solution ${ }^{7} v \in \mathcal{C}\left([0, T] ; \dot{B}_{2,1}^{\frac{N}{2}-1} \cap \dot{B}_{2,1}^{\frac{N}{2}-1+\alpha}\right) \cap L^{1}\left(0, T ; \dot{B}_{2,1}^{\frac{N}{2}+1} \cap \dot{B}_{2,1}^{\frac{N}{2}+1+\alpha}\right)$ on $[0, T] \times \mathbb{R}^{N}$.

There exists an $\epsilon_{0}>0$ such that for all $\epsilon \in\left(0, \epsilon_{0}\right)$ system $\left(N S C_{\epsilon}\right)$ has a unique solution $\left(b^{\epsilon}, u^{\epsilon}\right)$ with

$$
\begin{gathered}
u^{\epsilon} \in \mathcal{C}\left([0, T] ; \dot{B}_{2,1}^{\frac{N}{2}-1} \cap \dot{B}_{2,1}^{\frac{N}{2}+\alpha-1}\right) \cap L^{1}\left(0, T ; \dot{B}_{2,1}^{\frac{N}{2}+1} \cap \dot{B}_{2,1}^{\frac{N}{2}+\alpha+1}\right), \\
\epsilon b^{\epsilon} \in \mathcal{C}\left([0, T] ; \dot{B}_{2,1}^{\frac{N}{2}} \cap \dot{B}_{2,1}^{\frac{N}{2}+\alpha}\right) \quad \text { and } b^{\epsilon} \in L^{2}\left(0, T ; \dot{B}_{2,1}^{\frac{N}{2}} \cap \dot{B}_{2,1}^{\frac{N}{2}+\alpha}\right) \cap \mathcal{C}\left([0, T] ; \dot{B}_{2,1}^{\frac{N}{2}-1} \cap \dot{B}_{2,1}^{\frac{N}{2}+\alpha-1}\right)
\end{gathered}
$$

uniformly with respect to $\epsilon$.

Besides, the incompressible part of the velocity, $\mathcal{P} u^{\epsilon}$, tends to $v$ in

$$
\mathcal{C}\left([0, T] ; \dot{B}_{2,1}^{\frac{N}{2}-1} \cap \dot{B}_{2,1}^{\frac{N}{2}+\alpha-1}\right), \cap L^{1}\left(0, T ; \dot{B}_{2,1}^{\frac{N}{2}+1} \cap \dot{B}_{2,1}^{\frac{N}{2}+\alpha+1}\right)
$$

whereas $|D|^{\alpha-1+\frac{1}{p}}\left(b^{\epsilon}, \mathcal{Q} u^{\epsilon}\right)$ tends to 0 in $L^{p}\left(0, T ; L^{\infty}\right)$ with the rate $\epsilon^{\frac{1}{p}}$ whenever $p \geq 2$ if $N \geq 4, p>2$ if $N=3$.

In the case $N=2$ and under the assumptions made in the theorem above (even if $\alpha=0$ in fact), the incompressible solution $v$ is always global. We end up with the following statement:

Theorem 4.3. Let $\alpha \in(0,1 / 6], b_{0} \in \dot{B}_{2,1}^{0} \cap \dot{B}_{2,1}^{1+\alpha}, u_{0} \in \dot{B}_{2,1}^{0} \cap \dot{B}_{2,1}^{\alpha}$ and $f \in L^{1}\left(\mathbb{R}^{+} ; \dot{B}_{2,1}^{0} \cap \dot{B}_{2,1}^{\alpha}\right)$.

The incompressible system $(N S I)$ with initial datum $\mathcal{P} u_{0}$ and external force $\mathcal{P} f$ has a global solution $v \in$ $\mathcal{C}\left(\mathbb{R}^{+} ; \dot{B}_{2,1}^{0} \cap \dot{B}_{2,1}^{\alpha}\right) \cap L^{1}\left(\mathbb{R}^{+} ; \dot{B}_{2,1}^{2} \cap \dot{B}_{2,1}^{2+\alpha}\right)$. Besides, there exists an $\epsilon_{0}>0$ such that for all $\epsilon \in\left(0, \epsilon_{0}\right)$, system $\left(N S C_{\epsilon}\right)$ has a unique global solution which belongs to the space described in Theorem 4.2 with $T=+\infty$. In particular, $\mathcal{P} u^{\epsilon}$ tends to $v$ in $L^{\infty}\left(\mathbb{R}^{+} ; \dot{B}_{2,1}^{0} \cap \dot{B}_{2,1}^{\alpha}\right) \cap L^{1}\left(\mathbb{R}^{+} ; \dot{B}_{2,1}^{2} \cap \dot{B}_{2,1}^{2+\alpha}\right)$ whereas $|D|^{\alpha-\frac{3}{4}}\left(b^{\epsilon}, \mathcal{Q} u^{\epsilon}\right)$ tends to 0 in $L^{4}\left(\mathbb{R}^{+} ; L^{\infty}\right)$ with the rate $\epsilon^{\frac{1}{4}}$.

\subsection{Sketch of the proof}

Let us describe briefly the main steps of the proof. To avoid technicalities, we assume that $N \geq 4$ and $\alpha=1 / 2$ so that inequality (4) may be used. The reader is referred to [7] for the general case $N \geq 2$ and $\alpha>0$.

Step 1 (local well-posedness for fixed $\epsilon$ ). Under the assumptions of Theorem 4.2, it has been proved in [5] that there exists a positive $T_{\epsilon}$ such that $\left(N S C_{\epsilon}\right)$ has a unique solution $\left(b^{\epsilon}, u^{\epsilon}\right)$ on $\left[0, T_{\epsilon}\right] \times \mathbb{R}^{N}$ which belongs to the space $E_{T_{\epsilon}}^{\epsilon}$ defined as follows:

$$
\begin{aligned}
E_{T}^{\epsilon} \stackrel{\text { def }}{=}\left(L^{1}\left(0, T ; \widetilde{B}_{\epsilon \nu}^{\frac{N}{2}, 1} \cap \widetilde{B}_{\epsilon \nu}^{\frac{N}{2}+\frac{1}{2}, 1}\right) \cap \mathcal{C}\left([0, T] ; \widetilde{B}_{\epsilon \nu}^{\frac{N}{2}, \infty} \cap \widetilde{B}_{\epsilon \nu}^{\frac{N}{2}+\frac{1}{2}, \infty}\right)\right) \\
\quad \times\left(L^{1}\left(0, T ; \dot{B}_{2,1}^{\frac{N}{2}+1} \cap \dot{B}_{2,1}^{\frac{N}{2}+\frac{3}{2}}\right) \cap \mathcal{C}\left([0, T] ; \dot{B}_{2,1}^{\frac{N}{2}-1} \cap \dot{B}_{2,1}^{\frac{N}{2}-\frac{1}{2}}\right)\right)^{N} .
\end{aligned}
$$

Note however that the results of [5] do not supply a positive lower bound for $T_{\epsilon}$ when $\epsilon$ goes to 0 .

\footnotetext{
${ }^{7}$ It is well known that this assumption is always fulfilled for some positive $T$. Besides, one can take $T=+\infty$ if the norm of $\mathcal{P} u_{0}$ in $\dot{B}_{2,1}^{\frac{N}{2}-1}$ is small compared to $\mu$.
} 
Step 2 (uniform estimates for the solution). We use the fact that $\left(b^{\epsilon}, u^{\epsilon}\right)$ satisfies

$$
\left\{\begin{array}{l}
\partial_{t} b^{\epsilon}+u^{\epsilon} \cdot \nabla b^{\epsilon}+\frac{\operatorname{div} u^{\epsilon}}{\epsilon}=F^{\epsilon}, \\
\partial_{t} u^{\epsilon}+u^{\epsilon} \cdot \nabla u^{\epsilon}-\mathcal{A} u^{\epsilon}+\frac{\nabla b^{\epsilon}}{\epsilon}=G^{\epsilon},
\end{array}\right.
$$

with $F^{\epsilon}=-b^{\epsilon} \operatorname{div} u^{\epsilon}$ and $G^{\epsilon}=f+\frac{\epsilon \epsilon^{\epsilon}}{1+\epsilon b^{\epsilon}} \mathcal{A} u^{\epsilon}-k\left(\epsilon b^{\epsilon}\right) \nabla b^{\epsilon}$ where $k$ satisfies $k(0)=0$.

Up to the convection terms $u^{\epsilon} \cdot \nabla b^{\epsilon}$ and $u^{\epsilon} \cdot \nabla u^{\epsilon}$, this system is of type $\left(L_{\epsilon}\right)$. It is actually possible to extend inequality (3) to handle the convection terms (see [7]). Taking advantage of tame estimates, one eventually gets the following inequality for $s \in[N / 2, N / 2+1 / 2]$ :

$$
\begin{aligned}
\left\|b^{\epsilon}(t)\right\|_{\widetilde{B}_{\epsilon \nu}^{s, \infty}}+\left\|u^{\epsilon}(t)\right\|_{\dot{B}_{2,1}^{s-1}}+\int_{0}^{t}\left(\left\|b^{\epsilon}\right\|_{\widetilde{B}_{\epsilon \nu}^{s, 1}}+\left\|u^{\epsilon}\right\|_{\dot{B}_{2,1}^{s+1}}\right) \mathrm{d} \tau \leq \\
\quad C \mathrm{e}^{C V_{\epsilon}(t)}\left(\left\|b_{0}\right\|_{\widetilde{B}_{\epsilon \nu}^{s, \infty}}+\left\|u_{0}\right\|_{\dot{B}_{2,1}^{s-1}}+\int_{0}^{t}\left(\|f\|_{\widetilde{B}_{\epsilon \nu}^{s, \infty}}+\epsilon\left\|b^{\epsilon}\right\|_{L^{\infty}}\left\|u^{\epsilon}\right\|_{\dot{B}_{2,1}^{s+1}}\right) \mathrm{d} \tau\right)
\end{aligned}
$$

with

$$
V_{\epsilon}(t) \stackrel{\text { def }}{=} \int_{0}^{t}\left(\left\|u^{\epsilon}\right\|_{L^{\infty}}^{2}+\epsilon^{\frac{2}{3}}\left\|\nabla u^{\epsilon}\right\|_{L^{\infty}}^{\frac{4}{3}}+\left\|b^{\epsilon}\right\|_{L^{\infty}}^{2}\right) \mathrm{d} \tau
$$

Step 3. (convergence of the compressible part of the solution). Since $\left(\mathcal{Q} u^{\epsilon}, b^{\epsilon}\right)$ satisfies

$$
\left\{\begin{array}{c}
\partial_{t} b^{\epsilon}+\frac{\operatorname{div} \mathcal{Q} u^{\epsilon}}{\epsilon}=F^{\epsilon} \\
\partial_{t} \mathcal{Q} u^{\epsilon}+\frac{\nabla b^{\epsilon}}{\epsilon}=G^{\epsilon}
\end{array}\right.
$$

with

$$
F^{\epsilon}=-\operatorname{div}\left(b^{\epsilon} u^{\epsilon}\right) \quad \text { and } \quad G^{\epsilon}=-\nu \Delta \mathcal{Q} u^{\epsilon}+\mathcal{Q}\left(f+\frac{\mathcal{A} u^{\epsilon}}{1+\epsilon b^{\epsilon}}-k\left(\epsilon b^{\epsilon}\right) \nabla b^{\epsilon}-u^{\epsilon} \cdot \nabla u^{\epsilon}\right)
$$

Strichartz estimate (4) combined with various product estimates in Besov spaces lead to

$$
\left\|\left(b^{\epsilon}, \mathcal{Q} u^{\epsilon}\right)\right\|_{L_{t}^{2}\left(L^{\infty}\right)} \leq C \epsilon^{\frac{1}{2}}\left(\left\|b_{0}\right\|_{\dot{B}_{2,1}^{\frac{N}{2}-\frac{1}{2}}}+\left\|u_{0}\right\|_{\dot{B}_{2,1}^{\frac{N}{2}-\frac{1}{2}}}+\|\mathcal{Q} f\|_{L_{t}^{1}\left(\dot{B}_{2,1}^{\frac{N}{2}}-\frac{1}{2}\right)}+\left\|\left(b^{\epsilon}, u^{\epsilon}\right)\right\|_{E_{t}^{\epsilon}}\left(1+\left\|\left(b^{\epsilon}, u^{\epsilon}\right)\right\|_{E_{t}^{\epsilon}}\right)\right) .
$$

Note that we expect the previous step to provide a uniform control on $\left\|\left(b^{\epsilon}, u^{\epsilon}\right)\right\|_{E_{T}^{\epsilon}}$. Hence $\left(\mathcal{Q} u^{\epsilon}, b^{\epsilon}\right)$ should tend to 0 in $L^{2}\left(0, T ; L^{\infty}\right)$ with the rate $\epsilon^{\frac{1}{2}}$.

Step 4. (convergence of the incompressible part of the solution). In order to study the convergence of $\mathcal{P} u^{\epsilon}$, one can use the fact that $w^{\epsilon} \stackrel{\text { def }}{=} \mathcal{P} u^{\epsilon}-v$ satisfies

$$
\left\{\begin{array}{l}
\partial_{t} w^{\epsilon}+\mathcal{P}\left(w^{\epsilon} \cdot \nabla v+u^{\epsilon} \cdot \nabla w^{\epsilon}\right)-\mu \Delta w^{\epsilon}=-\mathcal{P}\left(\mathcal{Q} u^{\epsilon} \cdot \nabla v+u^{\epsilon} \cdot \nabla \mathcal{Q} u^{\epsilon}+\frac{\epsilon b^{\epsilon}}{1+\epsilon b^{\epsilon}} \mathcal{A} u^{\epsilon}\right), \\
w_{\mid t=0}^{\epsilon}=0
\end{array}\right.
$$

Note that we expect the right-hand side to be small when $\epsilon$ goes to 0. Indeed, the first two terms contain $\mathcal{Q} u^{\epsilon}$ which, according to the previous step is of size $\epsilon^{\frac{1}{2}}$ in $L^{2}\left(0, T ; L^{\infty}\right)$ and the last term should also be small since it begins with an $\epsilon$.

More concretely, standard results for the heat equation (see e.g. Prop. 7.4 in [7]) yield:

$$
\left\|w^{\epsilon}\right\|_{F_{t}} \leq C \exp \left(C \int_{0}^{t}\left(\|v\|_{\dot{B}_{2,1}^{\frac{N}{2}+1}}+\left\|u^{\epsilon}\right\|_{\dot{B}_{2,1}^{\frac{N}{2}+1}}\right) \mathrm{d} \tau\right) \int_{0}^{t}\left\|\mathcal{Q} u^{\epsilon} \cdot \nabla v+u^{\epsilon} \cdot \nabla \mathcal{Q} u^{\epsilon}+\frac{\epsilon b^{\epsilon}}{1+\epsilon b^{\epsilon}} \mathcal{A} u^{\epsilon}\right\|_{\dot{B}_{2,1}^{\frac{N}{2}-1} \cap \dot{B}_{2,1}^{2}}^{\frac{N}{2}-\frac{1}{2}} \mathrm{~d}
$$


with

$$
F_{t} \stackrel{\text { def }}{=}\left(L^{1}\left(0, t ; \dot{B}_{2,1}^{\frac{N}{2}+1} \cap \dot{B}_{2,1}^{\frac{N}{2}+\frac{3}{2}}\right) \cap \mathcal{C}\left([0, t] ; \dot{B}_{2,1}^{\frac{N}{2}-1} \cap \dot{B}_{2,1}^{\frac{N}{2}-\frac{1}{2}}\right)\right)^{N} .
$$

At a price of a bit of paradifferential calculus, it is possible to show that the right-hand side is indeed small. For instance, one has

$$
\left\|\mathcal{Q} u^{\epsilon} \cdot \nabla v\right\|_{L_{T}^{1}\left(\dot{B}_{2,1}^{\frac{N}{2}-1}\right)} \leq C \epsilon^{\frac{1}{2 N}}\left(\left\|\mathcal{Q} u^{\epsilon}\right\|_{L_{T}^{2}\left(\dot{B}_{2,1}^{\frac{N}{2}}\right)}+\epsilon^{-\frac{1}{2}}\left\|\mathcal{Q} u^{\epsilon}\right\|_{L_{T}^{2}\left(L^{\infty}\right)}\right) .
$$

Hence one can expect $w^{\epsilon}$ to tend to zero, or in other words, $\mathcal{P} u^{\epsilon}$ to tend to $v$.

Step 5. (bootstrap and continuation argument). In this last step, one combines the different estimates obtained hitherto and a standard bootstrap argument to prove that if $v \in F_{T}$ is a solution to (NSI) on $[0, T] \times \mathbb{R}^{N}$ then $\left(b^{\epsilon}, u^{\epsilon}\right)$ may be continued beyond $T$ for small enough $\epsilon$ (in other word, one can take $T_{\epsilon} \geq T$ ). Since the various estimates proved in the previous steps do not depend on $T$, the bootstrap argument still works if $T=+\infty$, yielding a global solution for $\left(N S C_{\epsilon}\right)$ with small $\epsilon$.

\section{ThE INCOMPRESSIBLE LIMIT IN THE PERIODIC CASE}

\subsection{The filtering method}

Prescribing periodic boundary conditions precludes from using dispersive properties in order to pass to the limit in $\left(N S C_{\epsilon}\right)$. Indeed, there is no chance that the acoustic waves go at infinity. Hence, there could be resonances which may hinder the convergence to the incompressible model.

We are going to see however that these resonances are very few and that the filtering method (introduced by Schochet in the framework of inviscid compressible fluids, see [28]) enables us to prove convergence toward the homogeneous incompressible Navier-Stokes equations.

In order to introduce the filtering method, let us rewrite $\left(N S C_{\epsilon}\right)$ as

$$
\frac{\mathrm{d}}{\mathrm{d} t}\left(\begin{array}{c}
b^{\epsilon} \\
u^{\epsilon}
\end{array}\right)+\frac{L}{\epsilon}\left(\begin{array}{c}
b^{\epsilon} \\
u^{\epsilon}
\end{array}\right)=\left(\begin{array}{l}
-\operatorname{div}\left(b^{\epsilon} u^{\epsilon}\right) \\
f-u^{\epsilon} \cdot \nabla u^{\epsilon}-\kappa b^{\epsilon} \nabla b^{\epsilon}-\widetilde{K}\left(\epsilon b^{\epsilon}\right) b^{\epsilon} \nabla b^{\epsilon}-I\left(\epsilon b^{\epsilon}\right) \mathcal{A} u^{\epsilon}
\end{array}\right)
$$

where the skewsymmetric operator $L$ is defined by

$$
L\left(\begin{array}{l}
b \\
u
\end{array}\right) \stackrel{\text { def }}{=}\left(\begin{array}{c}
\operatorname{div} u \\
\nabla b
\end{array}\right)
$$

$P^{\prime}(1+z) /(1+z)=1+\kappa z+z \widetilde{K}(z)$ with $K(0)=0$ and $I(z) \stackrel{\text { def }}{=} z /(1+z)$.

In the next proposition, we summarize the important properties of $L$ :

Proposition 5.1. The operator $L$ generates a unitary group $\mathrm{e}^{\tau L}$. Besides,

$$
\operatorname{Ker} L=\{(b, u) \mid b=\text { cste } \quad \text { and } \quad \operatorname{div} u=0\},
$$

and thus, $\mathrm{e}^{\tau L}\left(\begin{array}{c}0 \\ \mathcal{P} u\end{array}\right)=\left(\begin{array}{c}0 \\ \mathcal{P} u\end{array}\right)$.

For $Z: \mathbb{T}^{N} \rightarrow \mathbb{R}^{N+1}$, we denote by $\left(\mathrm{e}^{\tau L} Z\right)_{1}$ the first component of $\mathrm{e}^{\tau L} Z$ and by $\left(\mathrm{e}^{\tau L} Z\right)_{2}$ the last $N$ components of $\mathrm{e}^{\tau L} Z$. 
Let $V^{\epsilon} \stackrel{\text { def }}{=} \mathrm{e}^{\frac{t}{\epsilon} L}\left(\begin{array}{c}b^{\epsilon} \\ \mathcal{Q} u^{\epsilon}\end{array}\right)$. Remark that with the above convention, we have $b^{\epsilon}=\left(\mathrm{e}^{-\frac{t}{\epsilon} L} V^{\epsilon}\right)_{1}$ and $\mathcal{Q} u^{\epsilon}=$ $\left(\mathrm{e}^{-\frac{t}{\epsilon} L} V^{\epsilon}\right)_{2}$. Hence the new function $V^{\epsilon}$ satisfies

$$
\partial_{t} V^{\epsilon}+\mathcal{Q}_{1}^{\epsilon}\left(\mathcal{P} u^{\epsilon}, V^{\epsilon}\right)+\mathcal{Q}_{2}^{\epsilon}\left(V^{\epsilon}, V^{\epsilon}\right)-\nu \mathcal{A}_{2}^{\epsilon}(D) V^{\epsilon}=\mathrm{e}^{\frac{t}{\epsilon} L}\left(\begin{array}{c}
0 \\
\mathcal{Q}\left(f-\mathcal{P} u^{\epsilon} \cdot \nabla \mathcal{P} u^{\epsilon}\right)
\end{array}\right)+o(1)
$$

with $\mathcal{A}_{2}^{\epsilon}(D) B=\mathrm{e}^{\frac{t}{\epsilon} L}\left(\begin{array}{c}0 \\ \Delta\left(\mathrm{e}^{-\frac{t}{\epsilon} L} B\right)_{2}\end{array}\right)$,

$$
\begin{gathered}
\mathcal{Q}_{1}^{\epsilon}(u, B)=\mathrm{e}^{\frac{t}{\epsilon} L}\left(\begin{array}{c}
\operatorname{div}\left(u\left(\mathrm{e}^{-\frac{t}{\epsilon} L} B\right)_{1}\right) \\
\mathcal{Q}\left(u \cdot \nabla\left(\mathrm{e}^{-\frac{t}{\epsilon} L} B\right)_{2}+\left(\mathrm{e}^{-\frac{t}{\epsilon} L} B\right)_{2} \cdot \nabla u\right)
\end{array}\right), \\
\mathcal{Q}_{2}^{\epsilon}(A, B)=\frac{1}{2} \mathrm{e}^{\frac{t}{\epsilon} L}\left(\begin{array}{c}
\operatorname{div}\left(\left(\mathrm{e}^{-\frac{t}{\epsilon} L} A\right)_{1}\left(\mathrm{e}^{-\frac{t}{\epsilon} L} B\right)_{2}+\left(\mathrm{e}^{-\frac{t}{\epsilon} L} A\right)_{2}\left(\mathrm{e}^{-\frac{t}{\epsilon} L} B\right)_{1}\right) \\
\nabla\left(\left(\mathrm{e}^{-\frac{t}{\epsilon} L} A\right)_{2} \cdot\left(\mathrm{e}^{-\frac{t}{\epsilon} L} B\right)_{2}\right)+\kappa \nabla\left(\left(\mathrm{e}^{-\frac{t}{\epsilon} L} A\right)_{1}\left(\mathrm{e}^{-\frac{t}{\epsilon} L} B\right)_{1}\right)
\end{array}\right) .
\end{gathered}
$$

As we expect them to be negligible for vanishing $\epsilon$, we denoted by $o(1)$ the terms induced by $\widetilde{K}\left(\epsilon b^{\epsilon}\right) b^{\epsilon} \nabla b^{\epsilon}$ and $I\left(\epsilon b^{\epsilon}\right) \mathcal{A} u^{\epsilon}$.

The operators $\mathcal{A}_{2}^{\epsilon}(D), \mathcal{Q}_{1}^{\epsilon}$ and $\mathcal{Q}_{2}^{\epsilon}$ may be computed explicitly by mean of Fourier series (see [6]). After a cumbersome calculation that we prefer to avoid here, one obtains that $\mathcal{A}_{2}^{\epsilon}(D) B$ tends formally to $-\Delta B / 2$, and that the operators $\mathcal{Q}_{1}^{\epsilon}$ and $\mathcal{Q}_{2}^{\epsilon}$ tend to some first order bilinear operators $\mathcal{Q}_{1}$ and $\mathcal{Q}_{2}$. If $\mathcal{P} u^{\epsilon}$ tends to some limit $v$, the stationary phase theorem insures that the right-hand side of (7) tends to 0 in the sense of the distributions.

Finally, one can thus expect $\left(\mathcal{P} u^{\epsilon}, V^{\epsilon}\right)$ to tend to some limit $(v, V)$ with $v$ a solution to the incompressible Navier-Stokes equation

$$
\left\{\begin{array}{l}
\partial_{t} v+\mathcal{P}(v \cdot \nabla v)-\mu \Delta v=\mathcal{P} f \\
v_{\mid t=0}=\mathcal{P} u_{0}
\end{array}\right.
$$

and $V$ satisfying

$$
\left\{\begin{array}{l}
\partial_{t} V+\mathcal{Q}_{1}(v, V)+\mathcal{Q}_{2}(V, V)-\frac{\nu}{2} \Delta V=0 \\
V_{\mid t=0}=\left(b_{0}, \mathcal{Q} u_{0}\right)
\end{array}\right.
$$

At first sight and, up to the term $\mathcal{Q}_{1}(v, V)$ (which is linear with respect to $v$ ), this latter system resembles Navier-Stokes equations. Indeed the quadratic term $\mathcal{Q}_{2}(V, V)$ is first order. However it has been observed by $\mathrm{N}$. Masmoudi in [24], that, contrary to $v \cdot \nabla v$, the term $\mathcal{Q}_{2}(V, V)$ is so sparse that the diffusion $-\frac{\nu}{2} \Delta V$ dominates. Hence $V$ exists as long as $v$ is defined. Besides, one can prove that the term $\mathcal{Q}_{1}(v, V)$ does not contribute to the energy estimates. 


\subsection{Results}

In [6], we chose to use Sobolev spaces rather than Besov spaces ${ }^{8}$.

Let us first introduce the functional spaces needed in our main convergence result. Let $T \in(0, \infty]$. The existence of a solution $\left(b^{\epsilon}, u^{\epsilon}\right)$ will be proved in ${ }^{9}$

$$
\widetilde{E}_{T, \eta}^{s} \stackrel{\text { def }}{=}\left\{(b, u) \in \widetilde{C}_{T}\left(\widetilde{H}_{\eta}^{s, \infty} \times\left(H^{s-1}\right)^{N}\right) \mid b \in \widetilde{L}_{T}^{1}\left(\widetilde{H}_{\eta}^{s, 1}\right) \quad \text { and } \quad \nabla u \in\left(\widetilde{L}_{T}^{1}\left(H^{s}\right)\right)^{N^{2}}\right\}
$$

for some $s>N / 2$ and $\eta=\epsilon \nu$, whereas the incompressible solution $v$ belongs to

$$
\widetilde{F}_{T}^{s} \stackrel{\text { def }}{=}\left\{v \in\left(\widetilde{C}_{T}\left(H^{s-1}\right)\right)^{N} \mid \nabla u \in\left(\widetilde{L}_{T}^{1}\left(H^{s}\right)\right)^{N^{2}}\right\}
$$

Let us also define the following subspace of $\widetilde{F}_{T}^{s}$ :

$$
\widetilde{G}_{T}^{s} \stackrel{\text { def }}{=}\left\{v \in\left(\widetilde{C}_{T}\left(H^{s-1}\right)\right)^{N} \mid \nabla u \in\left(L_{T}^{2}\left(H^{s-1}\right)\right)^{N^{2}}\right\}
$$

Above, for $X=\widetilde{H}_{\eta}^{s, 1}$ or $H^{s}$, we denoted by $\widetilde{L}_{T}^{1}(X)$ a superspace of $L^{1}(0, T ; X)$ which is "almost" equal to $L^{1}(0, T ; X)$. The notation $\widetilde{C}_{T}(X)$ stands for a "large" subspace of $\mathcal{C}([0, T] ; X)$ (or of $\mathcal{C}\left(\mathbb{R}^{+} ; X\right) \times L^{\infty}\left(\mathbb{R}^{+} ; X\right)$ if $T=+\infty)$. The precise definitions may be found in [6].

One can now state our main result of convergence in the periodic case:

Theorem 5.2. Let $\alpha>0, b_{0} \in H^{\frac{N}{2}+\alpha}$ with zero average, $u_{0} \in H^{\frac{N}{2}+\alpha-1}$ and $f \in L^{1}\left(\mathbb{R}^{+} ; H^{\frac{N}{2}-1+\alpha}\right)$ with $\operatorname{div} f=0^{10}$. Assume that the incompressible Navier-Stokes equations with data $\mathcal{P} u_{0}$ and $f$ have a strong solution $v \in \widetilde{F}_{T}^{\frac{N}{2}+\alpha}$ on the time interval $[0, T]$ for some $T \in(0,+\infty]$. Then also

$$
\left\{\begin{array}{l}
\partial_{t} V+\mathcal{Q}_{1}(v, V)+\mathcal{Q}_{2}(V, V)-\frac{\nu}{2} \Delta V=0 \\
V_{\mid t=0}=\left(b_{0}, \mathcal{Q} u_{0}\right)
\end{array}\right.
$$

has a strong solution $V \in \widetilde{F}_{T}^{\frac{N}{2}+\alpha}$ on $[0, T]$.

Besides, there exists an $\epsilon_{0}>0$ such that for all $\epsilon \in\left(0, \epsilon_{0}\right)$, system $\left(N S C_{\epsilon}\right)$ has a strong solution $\left(b^{\epsilon}, u^{\epsilon}\right) \in \widetilde{E}_{T, \epsilon \nu}^{\frac{N}{2}+\alpha}$ on $[0, T]$ uniformly in $\epsilon^{11}$.

Moreover, $\mathcal{P} u^{\epsilon}-v=\mathcal{O}\left(\epsilon^{\frac{\alpha}{1+\alpha}}\right)$ in $\widetilde{F}_{T}^{\frac{N}{2}+\alpha}$ and $V^{\epsilon}$ tends to $V$ in $\widetilde{G}_{T}^{\frac{N}{2}+\alpha^{\prime}}$ for all $\alpha^{\prime}<\alpha$.

Remark 5.3. Let us stress that the above result holds true for $T=+\infty$. This is of particular interest if $N=2$ since $(N S I)$ has a global solution.

Remark 5.4. Theorem 5.2 actually states that

$$
\left(b^{\epsilon}, u^{\epsilon}\right)=(0, v)+\mathrm{e}^{-\frac{t}{\epsilon} L} V+o(1) \quad \text { in } \cap_{\alpha^{\prime}<\alpha} \widetilde{G}_{T}^{\frac{N}{2}+\alpha^{\prime}}
$$

Owing to the appearance of small divisors when proving the convergence of $V^{\epsilon}$, the exact meaning of $o(1)$ strongly depends on the quotients of the lengths of the periodic box $\mathbb{T}_{a}^{N}$. For special values of $\left(a_{1}, \cdots, a_{n}\right)$, the convergence may be slower than any power of $\epsilon$.

\footnotetext{
8 The only reason why is that Sobolev spaces are more "popular". It goes without saying that similar results may be obtained in Besov spaces.

${ }^{9}$ See remark 3.1 for the definition of $\widetilde{H}_{\eta}^{s, 1}$ and $\widetilde{H}_{\eta}^{s, \infty}$.

10 This assumption may be weakened, see [6].

11 So that in particular $b^{\epsilon} \in L^{2}\left(0, T ; H^{\frac{N}{2}+\alpha}\right) \cap \mathcal{C}\left([0, T] ; H^{\frac{N}{2}+\alpha-1}\right) \quad$ and $\quad \epsilon b^{\epsilon} \in \mathcal{C}\left([0, T] ; H^{\frac{N}{2}+\alpha}\right)$ uniformly.
} 


\subsection{Sketch of the proof}

Let us briefly describe the main steps of the proof:

Step 0. (global existence for $(L S))$.

We first have to show that if $(N S I)$ with data $\mathcal{P} u_{0}$ and $f$ has a solution $v \in \widetilde{F}_{T}^{\frac{N}{2}+\alpha}$ then $(L S)$ has also a solution $V \in \widetilde{F}_{T}^{\frac{N}{2}+\alpha}$. This fact has been discovered by N. Masmoudi in [24] for gradient-like data $V_{0}$ in $L^{2}$ or in $H^{\sigma}$ with $\sigma>N / 2$, then generalized to every Sobolev space $H^{\sigma}$ or Besov space $\dot{B}_{2,1}^{\sigma}$ with nonnegative regularity index.

The proof in the Sobolev case relies on simple energy estimates. Indeed, from $(L S)$, we readily get:

$$
\frac{1}{2} \frac{\mathrm{d}}{\mathrm{d} t}\|V\|_{H^{\sigma}}^{2}+\frac{\nu}{2}\|\nabla V\|_{H^{\sigma}}^{2}+\left(\mathcal{Q}_{1}(v, V) \mid V\right)_{H^{\sigma}}+\left(\mathcal{Q}_{2}(V, V) \mid V\right)_{H^{\sigma}}=0
$$

Now, it may be proved that $V$ is orthogonal to $\mathcal{Q}_{1}(v, V)$ for the inner product in $H^{\sigma}$. It turns out that $V$ is also orthogonal to $\mathcal{Q}_{2}(V, V)$ in $L^{2}$ (but not in $H^{\sigma}$ for $\sigma>0$ though). Hence, we get the same energy equality as for the usual incompressible Navier-Stokes equation with diffusion $\nu / 2$, namely:

$$
\|V(t)\|_{L^{2}}^{2}+\nu \int_{0}^{t}\|\nabla V(\tau)\|_{L^{2}}^{2} \mathrm{~d} \tau=\left\|V_{0}\right\|_{L^{2}}^{2} .
$$

As regards estimates for higher Sobolev regularity, the first order bilinear operator $\mathcal{Q}_{2}$ turns out to be far milder than the convection term $v \cdot \nabla v$ in $(N S I)$. As a matter of fact, it is so sparse as to behave in the Sobolev estimates as if it were one-dimensional: we have for all $\sigma \geq 0$ :

$$
\begin{aligned}
\left\|\mathcal{Q}_{2}(V, W)\right\|_{H^{\sigma}} & \leq C\left(\|V\|_{B_{2,1}^{\frac{1}{2}}}\|W\|_{H^{\sigma}}+\|W\|_{B_{2,1}^{\frac{1}{2}}}\|V\|_{H^{\sigma}}\right), \\
& \leq C\left(\|V\|_{H^{1}}\|W\|_{H^{\sigma}}+\|W\|_{H^{1}}\|V\|_{H^{\sigma}}\right) .
\end{aligned}
$$

Note that this is exactly the inequality we would have if $\mathcal{Q}_{2}(V, W)$ were equal to $\partial_{z}(V W)$ with $V$ and $W$ defined on the circle!

Now, combining with (8), we eventually get

$$
\|V\|_{L_{T}^{\infty}\left(H^{\sigma}\right)}+\nu\|\nabla V\|_{L_{T}^{2}\left(H^{\sigma}\right)} \leq C\left\|V_{0}\right\|_{H^{\sigma}} \mathrm{e}^{\frac{C}{\nu^{2}}\left\|V_{0}\right\|_{L^{2}}^{2}} .
$$

Endowed with this latter estimate, it is now easy to prove that $V$ is defined as long as $v$ exists.

Step 1. (local well-posedness for fixed $\epsilon$ ). This step is actually the same as in the whole space case. Since, we now want to use Sobolev spaces, it is only a matter of adapting Theorem 4.2 in [5] to the Sobolev spaces setting. Under the assumptions of Theorem 5.2, we get a unique solution $\left(b^{\epsilon}, u^{\epsilon}\right) \in \widetilde{E}_{T_{\epsilon}, \epsilon \nu}^{\frac{N}{2}+\alpha}$ of $\left(N S C_{\epsilon}\right)$ on some non trivial time interval $\left[0, T_{\epsilon}\right]$ which may depend on $\epsilon$.

Step 2. (uniform estimates for the solution). This step is also the same as in the whole space case. The only difference is that, due to the use of Sobolev spaces, we have to adapt (5) to the Sobolev setting (see Prop. 6.1 in [6]). Of course, the proof still relies on the study of the linearized system $\left(L_{\epsilon}\right)$.

Step 3. (convergence of the compressible part of the solution). Subtracting system $(L S)$ from $(7)$, we get the following equation for $z^{\epsilon} \stackrel{\text { def }}{=} V^{\epsilon}-V$ :

$$
\partial_{t} z^{\epsilon}-\frac{\nu}{2} \Delta z^{\epsilon}=\mathcal{Q}_{1}^{\epsilon}\left(v, z^{\epsilon}\right)+2 \mathcal{Q}_{2}^{\epsilon}\left(V, z^{\epsilon}\right)+\mathcal{Q}_{2}^{\epsilon}\left(z^{\epsilon}, z^{\epsilon}\right)+F\left(v, \mathcal{P} u^{\epsilon}, V^{\epsilon}, w^{\epsilon}\right)+R^{\epsilon}+o(1)
$$


where for fixed $(a, b, c)$, the function $d \mapsto F(a, b, c, d)$ is linear and continuous on Ker $L$ with a norm which may be bounded uniformly when $(a, b, c)$ belong to a bounded subset of $\left(\widetilde{F}_{T}^{\frac{N}{2}+\alpha}\right)^{3}, w^{\epsilon}=\mathcal{P} u^{\epsilon}-v$ and

$$
R^{\epsilon}=\left(\mathcal{Q}_{1}-\mathcal{Q}_{1}^{\epsilon}\right)(v, V)+\left(\mathcal{Q}_{2}-\mathcal{Q}_{2}^{\epsilon}\right)(V, V)+\nu\left(\mathcal{A}_{2}^{\epsilon}(D)-\frac{\Delta}{2}\right) V^{\epsilon}+\mathrm{e}^{\frac{t}{\epsilon} V}\left(\begin{array}{c}
0 \\
f-\mathcal{Q}(v \cdot \nabla v)
\end{array}\right) .
$$

Let us give a rough analysis of the right-hand side of the equation satisfied by $z^{\epsilon}$.

The first two terms are first order and linear in $z^{\epsilon}$, hence, after a suitable use of a Gronwall type argument, we expect them to generate a harmless exponential term in the estimates. For small $z^{\epsilon}$, the third term is clearly negligible.

Since the fourth term is linear in $w^{\epsilon}$ and uniformly bounded by virtue of step one, we predict him to be comparable to the size of $w^{\epsilon}$ (whose study is the object of step 4).

On the other hand, the term $R^{\epsilon}$ is unlikely to be small in any Sobolev space. Indeed, since the semi-group associated to $L$ is unitary, it is clear that the last term of $R^{\epsilon}$ has a Sobolev norm independent of $\epsilon$. In order to glean some smallness, one has to take advantage of the oscillating properties of $R^{\epsilon}$.

This may be done (after S. Schochet in [28]) by introducing the function $\phi_{M}^{\epsilon} \stackrel{\text { def }}{=} z^{\epsilon}+\epsilon \widetilde{R}_{M}^{\epsilon}$ where $\epsilon \widetilde{R}_{M}^{\epsilon}$ resembles a primitive of $R^{\epsilon}$ truncated at spatial frequencies smaller than some positive $M$ to be fixed hereafter. More precisely, denoting by $R_{M}^{\epsilon}$ the part of $R^{\epsilon}$ with frequencies less than $M$, we have

$$
\epsilon \partial_{t} \widetilde{R}_{M}^{\epsilon}=R_{M}^{\epsilon}+\epsilon \widehat{R}_{M}^{\epsilon}
$$

for some term $\widehat{R}_{M}^{\epsilon}$ which may be bounded in Sobolev spaces independently of $\epsilon$ provided we pay enough powers of $M$ for that.

For fixed $M$ and in the asymptotic of vanishing $\epsilon$, bounding $z^{\epsilon}$ amounts to bounding $\phi_{M}^{\epsilon}$. Let us point out however that, owing to the appearance of small divisors in the definition of $\widetilde{R}_{M}^{\epsilon}$ and $\widehat{R}_{M}^{\epsilon}$, the estimates that we are going to obtain for $z^{\epsilon}$ strongly depend on $M$. We thus have to optimize the choice of $M$ with respect of $\epsilon$. We eventually end up with an inequality of the type

$$
\left\|z^{\epsilon}\right\|_{\widetilde{G}_{T}^{\frac{N}{2}}} \leq \mathcal{K}\left(\left\|\left(b^{\epsilon}, u^{\epsilon}\right)\right\|_{\widetilde{E}_{T, \epsilon \nu}^{\frac{N}{2}+\alpha}},\|v\|_{\widetilde{F}_{T}^{\frac{N}{2}+\alpha}},\|V\|_{\widetilde{F}_{T}^{\frac{N}{2}+\alpha}}\right)\left(\left\|w^{\epsilon}\right\|_{\widetilde{F}_{T}^{\frac{N}{2}+\alpha}}+o(1)\right)
$$

with $\mathcal{K}$ a continuous positive function.

Step 4. (convergence of the incompressible part of the solution). Since $\mathcal{Q} u^{\epsilon} \cdot \nabla \mathcal{Q} u^{\epsilon}$ is a gradient, one easily gathers that $w^{\epsilon}$ satisfies

$$
\partial_{t} w^{\epsilon}-\mu \Delta w^{\epsilon}=-\mathcal{P}\left(A^{\epsilon} \cdot \nabla w^{\epsilon}+w^{\epsilon} \cdot \nabla A^{\epsilon}+w^{\epsilon} \cdot \nabla w^{\epsilon}+\mathcal{Q} u^{\epsilon} \cdot \nabla v+v \cdot \nabla \mathcal{Q} u^{\epsilon}+I\left(\epsilon b^{\epsilon}\right) \mathcal{A} u^{\epsilon}\right)
$$

with $A^{\epsilon} \stackrel{\text { def }}{=} v+\mathcal{Q} u^{\epsilon}$.

Note that the first two terms of the right-hand side are linear in $w^{\epsilon}$, hence harmless in the estimates as far as $A^{\epsilon}$ is uniformly bounded in a suitable Sobolev space (namely $L^{1}\left(0, T ; H^{\frac{N}{2}+1} \cap \operatorname{Lip}\right)$ ). The third term is quadratic in $w^{\epsilon}$ hence negligible for small $w^{\epsilon}$. The last term is also small since it contains a factor $\epsilon$.

On the one hand, the two terms $\mathcal{Q} u^{\epsilon} \cdot \nabla v$ and $v \cdot \nabla \mathcal{Q} u^{\epsilon}$ are unlikely to be small in any Sobolev space. Indeed, according to the previous step, we expect $\mathcal{Q} u^{\epsilon}$ to be asymptotically equivalent to $\mathrm{e}^{-\frac{t}{\epsilon} L} V$, hence to have Sobolev norms independent of $\epsilon$. On the other hand, we can take advantage from oscillating properties of $\mathcal{Q} u^{\epsilon}$. As a matter of fact, after performing a change of function $w^{\epsilon}$ similar to the one which has been done in the previous step for $z^{\epsilon}$, one ends up with an estimate of the type

$$
\left\|w^{\epsilon}\right\|_{\widetilde{F}_{T}^{\frac{N}{2}+\alpha}} \leq \mathcal{K}\left(\left\|\left(b^{\epsilon}, u^{\epsilon}\right)\right\|_{\widetilde{E}_{T, \epsilon \nu}^{\frac{N}{2}+\alpha}},\|v\|_{\widetilde{F}_{T}^{\frac{N}{2}+\alpha}},\|V\|_{\widetilde{F}_{T}^{\frac{N}{2}+\alpha}}\right)\left(\epsilon^{\frac{\alpha}{1+\alpha}}+\left\|w^{\epsilon}\right\|_{\widetilde{F}_{T}^{\frac{N}{2}+\alpha}}^{2}\right) .
$$


Step 5. (bootstrap and continuation argument). Since by assumption $(N S I)$ has a solution $v \in \widetilde{F}_{T}^{\frac{N}{2}+\alpha}$ on $[0, T]$, step 0 insures that $(L S)$ has also a solution $V \in \widetilde{F}_{T}^{\frac{N}{2}+\alpha}$ on $[0, T]$. According to step one, $\left(N S C_{\epsilon}\right)$ has a solution $\left(b^{\epsilon}, u^{\epsilon}\right) \in \widetilde{E}_{T_{\epsilon}, \epsilon \nu}^{\frac{N}{2}+\alpha}$ on some non trivial interval $\left[0, T_{\epsilon}\right]$.

Next, by combining the uniform estimates obtained in step 2 with (9) and (10), and assuming that $\epsilon$ is small enough, one obtains estimates for $\left(b^{\epsilon}, u^{\epsilon}\right)$ depending only on the data, on $v$ and on $V$ but independent of $\epsilon$ for small $\epsilon$. Standard bootstrap and continuation arguments then enable us to prove that $T^{\epsilon}$ may be chosen greater than or equal to $T$ and that the desired results of convergence are true.

\section{SOME OPEN PROBLEMS}

Let us end this survey with a short list of open problems related to the zero Mach number limit.

\subsection{Convergence results for data with critical regularity}

In the statements of Theorems 4.2 and 5.2, data are slightly smoother than required according to the scaling properties of $\left(N S C_{\epsilon}\right)$. For fixed $\epsilon$ however, it is possible to prove that $\left(N S C_{\epsilon}\right)$ is well-posed for a large class of data having critical regularity (see Th. 2.1).

As regards the zero Mach number limit, it turns out that some global in time results are available for $\left(N S C_{\epsilon}\right)$ in $\mathbb{R}^{N}$ or $\mathbb{T}_{a}^{N}$ under the condition that the data are small, namely

$$
\left\|b_{0}\right\|_{\dot{B}_{2,1}^{\frac{N}{2}-1}}+\mu\left\|b_{0}\right\|_{\dot{B}_{2,1}^{\frac{N}{2}}}+\left\|u_{0}\right\|_{\dot{B}_{2,1}^{\frac{N}{2}-1}}+\|f\|_{L^{1}\left(\dot{B}_{2,1}^{\frac{N}{2}}-1\right)} \ll \mu .
$$

The reader may refer to [7] and [6] for more details.

Whether a statement of convergence in the spirit of Theorems 4.2 and 5.2 may be proved for large data with critical regularity is open.

\subsection{The case of more general domains}

When comparing the cases $\mathbb{R}^{N}$ and $\mathbb{T}_{a}^{N}$, we notice that boundary conditions have a definite influence on the low Mach number limit for barotropic flows.

One can wonder now what happens for flows in more general domains. In the case of bounded domains with homogeneous Dirichlet boundary conditions on the velocity, some very exciting results have been obtained in [10] in the framework of global weak solutions. There, it has been discovered that the "quality" of the convergence depends on the shape of the domain in a way that seems related to Schiffer's conjecture for the Laplacian.

It would be interesting to study whether stronger solutions behave similarly as regards convergence in bounded domains. However, whether similar statements as Theorems 4.2 or 5.2 may be proved is open for at least technical reasons: working in bounded domains precludes Fourier analysis...

\subsection{Low Mach number limit for the full Navier-Stokes equations}

As regards well-posedness theory, there is no additional conceptual difficulty in considering the full NavierStokes system rather than the barotropic equations. As a matter of fact, local and global existence results for smooth data have been stated in [25], and the case of critical regularity has been investigated in $[4,5]$.

On the other hand, passing to the zero Mach number limit in the full system is much more involved. One of the reasons why is that the limit system is no longer the usual incompressible Navier-Stokes equations. One has to mention however that some preliminary very promising results have been obtained by Alazard in the polytropic case (see [1]). 


\section{REFERENCES}

[1] T. Alazard, Work in progress (2004).

[2] M. Cannone, Ondelettes, paraproduits et Navier-Stokes. Diderot Ed., Paris (1995).

[3] R. Danchin, Global existence in critical spaces for compressible Navier-Stokes equations. Invent. Math. 141 (2000) 579-614.

[4] R. Danchin, Global existence in critical spaces for flows of compressible viscous and heat-conductive gases. Arch. Rational Mech. Anal. 160 (2001) 1-39.

[5] R. Danchin, Local theory in critical spaces for compressible viscous and heat-conductive gases. Comm. Partial Differential Equations 26 (2001) 1183-1233.

[6] R. Danchin, Zero Mach number limit for compressible flows with periodic boundary conditions. Am. J. Math. 124 (2002) $1153-1219$

[7] R. Danchin, Zero Mach number limit in critical spaces for compressible Navier-Stokes equations. Ann. Sci. Éc. Norm. Sup. (2002).

[8] R. Danchin, On the uniqueness in critical spaces for compressible navier-stokes equations. Nonlinear Differential Equations and Applications, to appear (2002).

[9] B. Desjardins and E. Grenier, Low Mach number limit of viscous compressible flows in the whole space. Proc. Roy. Soc. London Ser. A, Math. Phys. Eng. Sci. 455 (1999) 2271-2279.

[10] B. Desjardins, E. Grenier, P.-L. Lions and N. Masmoudi, Incompressible limit for solutions of the isentropic Navier-Stokes equations with Dirichlet boundary conditions. J. Math. Pures Appl. (2002).

[11] H. Fujita and T. Kato, On the Navier-Stokes initial value problem. I. Arch. Rational Mech. Anal. 16 (1964) 269-315.

[12] I. Gallagher, A remark on smooth solutions of the weakly compressible periodic Navier-Stokes equations. J. Math. Kyoto Univ. 40 (2000) 525-540.

[13] J. Ginibre and G. Velo, Generalized Strichartz inequalities for the wave equation. J. Funct. Anal. 133 (1995) 50-68.

[14] T. Hagstrom and J. Lorenz, All-time existence of classical solutions for slightly compressible flows. SIAM J. Math. Anal. 29 (1998) 652-672.

[15] D. Hoff, The zero-Mach limit of compressible flows. Comm. Math. Phys. 192 (1998) 543-554.

[16] T. Kato, Strong $L^{p}$-solutions of the Navier-Stokes equation in $R^{m}$, with applications to weak solutions. Math. Z. 187 (1984) $471-480$.

[17] M. Keel and T. Tao, Endpoint Strichartz estimates. Am. J. Math. 120 (1998) 955-980.

[18] S. Klainerman and A. Majda, Compressible and incompressible fluids. Comm. Pure Appl. Math. 35 (1982) 629-651.

[19] H.-O. Kreiss, J. Lorenz and M.J. Naughton, Convergence of the solutions of the compressible to the solutions of the incompressible Navier-Stokes equations. Adv. Appl. Math. 12 (1991) 187-214.

[20] P.-L. Lions, Mathematical topics in fluid mechanics. Vol. 1: Incompressible models. Oxford Clarendon Press (1996).

[21] P.-L. Lions, Mathematical topics in fluid mechanics. Vol. 2: Compressible models. Oxford Clarendon Press (1998).

[22] P.-L. Lions and N. Masmoudi, Incompressible limit for a viscous compressible fluid. J. Math. Pures Appl. (9) 77 (1998) 585-627.

[23] P.-L. Lions and N. Masmoudi, Une approche locale de la limite incompressible. C. R. Acad. Sci. Paris (1999).

[24] N. Masmoudi, Incompressible, inviscid limit of the compressible Navier-Stokes system. Ann. Inst. H. Poincaré Anal. Non Linéaire (2001).

[25] A. Matsumura and T. Nishida, The initial value problem for the equations of motion of viscous and heat-conductive gases. J. Math. Kyoto Univ. 20 (1980) 67-104.

[26] G. Métivier and S. Schochet, The incompressible limit of the non-isentropic Euler equations. Arch. Rational Mech. Anal. 158 (2001) 61-90.

[27] G. Métivier and S. Schochet, Averaging theorems for conservative systems and the weakly compressible Euler equations. J. Differential Equations 187 (2003) 106-183.

[28] S. Schochet, Fast singular limits of hyperbolic PDEs. J. Differential Equations 114 (1994) 476-512.

[29] S. Ukai, The incompressible limit and the initial layer of the compressible Euler equation. J. Math. Kyoto Univ. 26 (1986) 323-331. 